\title{
A Study on Chemical Accident Emergency Plan Generating Techniques
}

\author{
Feng Liu*, Qiang Hu, Xiaojia Jiang, Yuping Sun, Junhui Pan, \\ Weiyin Zhang, Lei Yang \\ Institute of NBC Defence, Beijing 102205, China \\ *fliu_pku@sina.com
}

Keywords: chemical accident; emergency plan; plan generating system; plan matching.

Abstract. In this paper, techniques for chemical accident emergency generating were studied, and the basic framework of plan generating system was established through analysis of the contents of a typical emergency plan. Under this framework, the approaches of generating different parts of a plan were discussed and the plan matching techniques were studied.

\section{Introduction}

The chemical accidents are mostly reported to be toxic gas leaking. The toxic gas could contaminate the atmosphere in a large range after its diffusion in the atmosphere, which threat people's health and life. The government organs and chemical enterprises should attach importance to the chemical emergency response and make the corresponding emergency plan in advance.

At present, most of research on the chemical risk control regime goes to the accident emergency control. The decision maker will issue an alert, organize evacuation, rescue and relief after the chemical accident, which will be carried out under the guideline of the emergency plan. Currently, the major methods for determining the accident emergency plan are mainly case-based reasoning (CBR) [1] and rule-based reasoning (RBR) [2]. The former is to search the matching plan from the plan database; the latter is to form a new plan according to the expertise. Each method has its distinct advantages and shortcomings. CBR needs a huge plan database ahead of time to develop the matching algorithm; while RBR requires informative and practical inference rules to generate a new plan. The research combining the two methods are emerging in recent years [3]. The plans in both CBR and RBR are drawn from the cases and expertise, which doesn't take the accident hazard stimulation and quantitative information of risk assessment into consideration. In addition, the plans also need to be improved in order to make the scientific decision for mass evacuation, to realize the reasonable allocation and scheduling of rescue resources, to lower the hazard control cost and enhance the emergency response efficiency. Recent years have witnessed the progress of the theory and method. The techniques, like GIS positioning for accident source and real-time release of hazard range, are urged to have an emergency plan generating method matching these advanced method and technique.

\section{Structure of Chemical Accident Emergency Plan Generating}

The Basic Formation of Emergency Plan. The plan could be classified into general and special content in order to realize the intellectual generating of an emergency plan. The general content is shared by each plan, and it includes the purpose of plan preparation, the basis (relevant laws and regulations) and principles, which belongs to the pure text content. While the special content varies due to specific plans, according to different methods of their generating, the special content could also be divided into fixed content and variable content. Fixed content refers to the basic information of the chemical facility, e.g. geography, traffic, running system of the unit, type, characteristics and amount of chemicals, units in vicinity, community and eco-region, as well as emergency response resource, like the emergency response force of own unit and the surrounding rescue force can rely on. All of the above factors will not change with specific incident. The variable content will differ due to specific incident; which includes the degree and type of accident, atmospheric condition, hazard consequence 
assessment, emergency response measurement and allocation of the rescue resources. The basic formation of emergency plan is as Fig. 1.

$$
\begin{aligned}
& \text { Content of Chemical Accident Emergency Plan }
\end{aligned}
$$

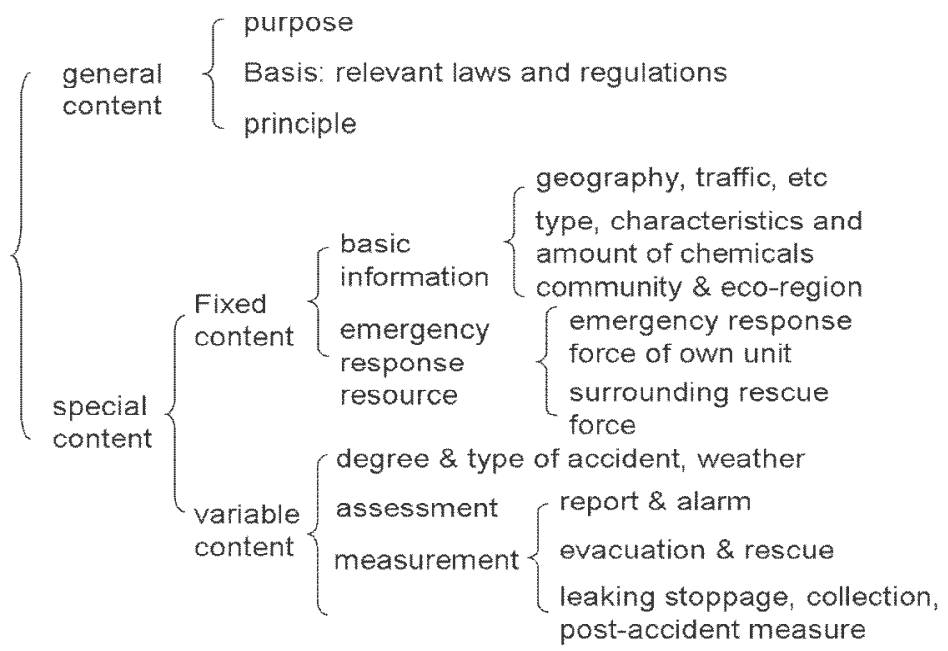

Fig. 1: basic parts of chemical emergency response plan

System Framework of Plan Generating. The intellectual generating method can automatically and batch-generate effective plans, and then the plans can form a plan database. The content of plan includes not only the conventional text content like personnel plan, monitoring plan, emergency rescue plan, disaster relief plan, decision-making assistance plan, but also the quantified evacuation range and route, the key factors of the plan like rescue force formation, and the visualized display of concentration, range, lasting time of chemical diffusion. One of the application of artificial intelligence theory reported in some research literatures, is that the automatically generation of emergency plan combined with graphics and text through the imitation of human reasoning process by computers [4, 5].

Plan Generating Method and Process. Research on the generating method according to the features of different parts in each plan must be worked to generate the emergency plan. See Fig. 2.

The general content is text part, which could be imported directly.

The fixed content includes each type of basic statistic, hence, the way of establishing a database in adapted to import information. The database includes basic information and emergency rescue resource databases, in which the statistics could be called to form a corresponding text plan. The database could also provide the statistics needed for the emergency risk assessment and emergency response planning.

The most difficult procedure is to generate the variable content, which need to generate an emergency plan based on chemical hazard assessment technique and chemical risk optimal control technique. Its basic process goes like: stimulate and assess the toxic gas diffusion range and the possible hazard caused by accident by incident hazard assessment model, in accordance with the metrological data and toxic gas source data; combine the chemical risk information monitored and stimulated, the emergency response force and appropriate countermeasures with expertise on rules applying, to generate a graft plan; and then, carry out deductive stimulation and assessment on the effect of the plan to test its feasibility; and as well as the test and perfect of the completeness of the rules. 


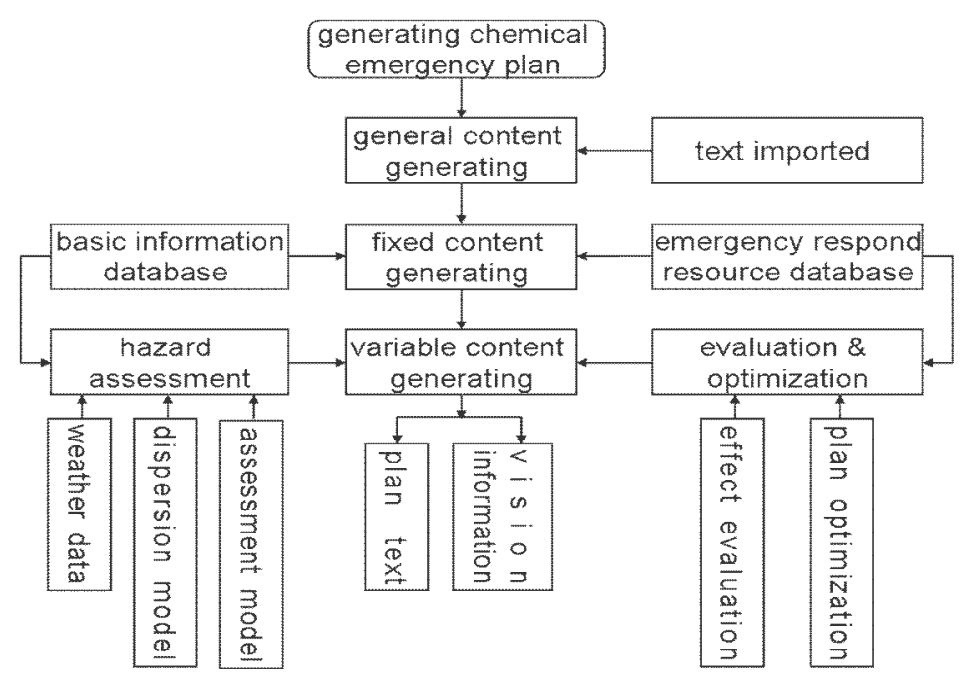

Fig. 2: generating process of chemical emergency response plan

The Optimization of the Plan. To stimulate, assess and release information combined with expertise by applying chemical hazard early warning system can generate a plan. For a same accident, although the plans can run the chemical accident risk, it will cost differently due to the apparently different plans made by different experts. Therefore, the most optimized plan should be selected, with the optimizing to the dominated factors to ensure the minimum cost of control. The dominated factors are: the range and route of evacuation, the alert time, and the use of protection, firefighting and rescue force. Different combinations lead to different cost. For instance, when the decision maker identify a relatively small evacuation range, the necessary countermeasures like control the risk source, leaking stoppage and decontamination needed to be carried out by responders, the cost of treatment will increase; if a low cost treatment is expected, a relatively larger evacuation range will be determined to ensure the safety of the mass, meanwhile the requirement for the risk source control will be lower. So, valuing different dominated factors is important to realize the optimized control.

The plan based on expertise has a strong subjectivity. For a same accident, it may give out multiple plans of countermeasure. Considering each countermeasure may have a variety of values, the number of the combination will be extremely huge. So, the optimization of the plan will be remarkable effected for the chemical emergency which can cause a huge property loss.

The optimization of the emergency countermeasure is different from the same issue on general controlling that is because the value of control variables is discrete. For example, the mobilization of fire engine and fire brigade, the function relation between control cost and control measure is usually segmented or discrete. We need to establish a reasonable cost function after a comprehensive economic measure, and then select an appropriate optimized algorithm.

\section{The Supporting Technique Research on Chemical Emergency Response Plan}

In order to guarantee the similarity between the plan and the real situation, the most matched method must be studied. Selecting the plan that is close to the real situation from the database, based on which the decision making organ can obtain the feasible emergency plan.

Formation of Pan Database. Through ranking the importance of the factors impacting the accident emergency response, e.g. source strength, toxicity, terrain, wind speed, wind direction and stability, the priority of each factor will be leveled based on the stimulation and assessment of plenty random set of accidents. According to the intellectual plan generating method, the factors will be leveled by their priorities, and will be valued to a certain interval within a reasonable scope. The optimized plans will be batch generated and form a plan database through the applying of chemical risk assessment software, based on the chemical accident degrees and the corresponding rules of countermeasures. The feature information could be matched with the plan in the database and choose a most matched plan when there is emergency happened. 
Express of Plan. The plan is the major objective of plan management system. The plans should be stored in computers in an easily-read and processed way before they were effectively managed. The express of plan is to transfer the plan into information which is easily read and stored by computer.

The plan is consisted of three parts:

(1) The overall description of the solving method, namely the description of the solution, is also known as condition description. The plan description can be categorized as subject attribute and feature attribute. Subject attribute refers to the basic information of the plan itself, e.g. type of the plan; feature attribute is the critical attribute which impact the accident greatly, e.g. the location of accident. The feature attribute is the key point for plan matching.

(2) The description of the solving method, namely the description of the solution, is also known as result description. The result description mainly refers to the description of public emergency response plan.

(3) The description of the post-accident effect, namely it is the assessment and conclusion of the emergency response.

Based on the content of the plan, quads can describe the plan. The quads can be interpreted as: plan $=<$ subject attribute, feature attribute, emergency response plan description, conclusion $>$, simplified as:

$$
\mathrm{Y}=\langle\mathrm{X} 1, \mathrm{X} 2, \mathrm{X} 3, \mathrm{X} 4\rangle
$$

Among them, $\mathrm{X} 1$ stands for the subject attribute of the plan, according to the real situation, $\mathrm{X} 1$ is a finite set; X2 refers to the feature information of the plan, this set must be a nonempty set for the retrieval and matching of the plan; X3 is the description of the emergency response plan; X4 is the assessment and conclusion of the whole emergency response.

Retrieval of Plan. The retrieval of plan is to extract the feature attribute of current accident, and search the matching plan in the plan database when there is a chemical emergency. Since the plan database is usually very huge, we can divide the database into several sub-databases according to the types of plans. Finding the corresponding sub-database ahead of time can narrow the searching scope. The consequence of retrieval is usually one or more plans which are close the current accident.

Two critical issues are involved in the retrieval: the one is the determination of weight coefficient of each factor; the other is the matching of similarity of each factor.

The Determination of Weight Coefficient of Each Factor. Each plan will involve several feature attributes, while each attribute's importance may differ. The improvement of the retrieval accuracy requires the determination of weight coefficient according to the relative importance of each feature attribute. The weight accuracy will influence the consequence of plan retrieval to a certain extent.

The method of weight determination varies, e.g. subjective assignment method, analytic hierarchy process, multi-objective optimal method, multi-objective maximum distance method, etc.

The Similarity Calculation of Plan. Actually, the plan matching is the comparison between the features of current accident and presupposed ones. The final consequence will be the most optimal matched plans by calculating the similarity.

The plan matching is neither the similarity of single attribute nor the sum of all the attributes, because a single plan may consist of many attributes. The consideration must be given to both the partial similarity of single attribute and the overall similarity of all attributes.

Adjustment of Plan. The adjustment of plan is to adjust the difference-existing feature attribute based on the differences between the current accident and the accident retrieved, in order to enable them to fulfill the current task. Transformational adaptation and parameter adjustment are usually adapted in plan adjustment, whose main concern is the difference between the case retrieved and emerging problems, and make the relevant adjustment.

Systematize the Study of Plan. The adjustment of plan is to adjust the difference-existing feature attribute based on the differences between the current accident and the accident retrieved, in order to enable them to fulfill the current task. Transformational adaptation and parameter adjustment are usually adapted in plan adjustment, whose main concern is the difference between the case retrieved and emerging problems, and make the relevant adjustment. 


\section{Conclusions}

Since the chemical accident is multi-typed, complicated conditioned, and hard to make targeted plan, to develop the emergency plan intellectual generating technique is the right way to focus on the practicability and efficiency of chemical emergency response. This paper establishes a basic framework of chemical emergency response plan, classifies the plan according to their characteristics and put forward corresponding generating method. The paper also further studies the plan matching technique, and suggests the realizing route for the plan express and plan retrieval.

\section{Acknowledgements}

This work was supported by National Natural Foundation of China (No. 41375154).

\section{References}

[1] Kolodner J. L. An introduction to case based reasoning. Artificial Intelligence Review, 6(1), pp. 3-34, 1992.

[2] Luo J., Liu Z. and Z. Shi. Analysis and realize of KCExpert system. Computer Project, 32 (2), pp. 232-233, 2006

[3] Luo J., Shi Z., He Q., et al. A CBR-RBR Combined Fast Plan Generating System. Study and Development of Computer , 44 (4), pp. 660-666, 2007

[4] Shi Z.: Advanced Artificial Intelligence. Science Press, Beijing 2006

[5] Chen L. Study on GIS-Based Contaminated Emergency Plan Automatically Generating in Lake Source. Dissertation of Nanjing Normal University, 2007 